$\mathrm{SINP} / \mathrm{TNP} / 02-11$

UMD-PP-02-045

\title{
A MINIMAL THREE GENERATION SEESAW SCENARIO FOR LSND
}

\author{
Biswajoy Brahmachari ${ }^{a},{\text { Sandhya } \text { Choubey }^{b} \text { and Rabindra N. Mohapatra }}^{c}$ \\ (a) Theoretical Physics Group, Saha Institute of Nuclear Physics, \\ 1/AF Bidhannagar, Kolkata 700064, INDIA \\ (b) Department of Physics and Astronomy, University of Southampton, \\ Highfield, Southampton S017 1BJ, UK \\ (c) Department of Physics and Astronomy, University of Maryland, \\ College Park, MD-20742, USA
}

\begin{abstract}
$\underline{\text { Abstract }}$
We show that in the minimal three generation seesaw models for neutrinos, the presence of leptonic $\left(L_{e}+L_{\mu}-L_{\tau}\right) \times S_{2}$ symmetry leads to one of the right handed neutrinos remaining massless. This state can then be identified with the sterile neutrino required for a simultaneous understanding of solar, atmospheric and LSND observations. We present a gauge model where the presence of higher dimensional operators originating from Planck scale physics lead to a realistic $2+2$ mixed scenario that fits all oscillation data. The model predicts a range for the mixing angle $U_{e 3}$ and an effective mass for neutrinos emitted in tritium decay, which can be used to test this model.
\end{abstract}




\section{Introduction}

The evidence for neutrino masses and mixings now appears to be quite solid from the positive results for neutrino oscillations from solar neutrino data in seven different experiments, Chlorine, Kamiokande, Super-Kamiokande, SAGE, GALLEX, GNO, SNO [1] and atmospheric neutrino data from Super-Kamiokande, IMB, Soudan and MACRO experiments [2]. The third piece of evidence is from the Los Alamos LSND experiment that shows an oscillation from the muon neutrino to the electron neutrino (from $\overline{\nu_{\mu}}$ to $\overline{\nu_{e}}$ ) [3]. The KARMEN $\left[4\right.$ experiment which looked for $\overline{\nu_{\mu}}$ to $\overline{\nu_{e}}$ oscillation did not find any evidence for it and eliminated a large fraction of the parameter space allowed by LSND. It is hoped that the Mini-BOONE experiment at FERMILAB [5] will settle the issue in near future.

Theoretical analyses have made it clear that a simultaneous explanation of all the neutrino oscillation observations requires the existence of an ultralight sterile neutrino in addition to the three known active neutrinos $\left(\nu_{e}, \nu_{\mu}, \nu_{\tau}\right)$ [6]. There are two mass patterns that are currently under active discussion; the $2+2$ scheme[6] and $3+1$ scheme[7]. The $2+2$ scheme has two mass eigenstate neutrinos with mass around an $\mathrm{eV}$ and a lighter pair with mass near $10^{-3} \mathrm{eV}$, with the latter pair explaining the solar neutrino data, the former explaining the atmospheric neutrino data and the gap between the two pairs explaining the LSND results. Recent SNO data disfavors the original version of the $2+2$ model where all the missing solar $\nu_{e}$ 's are converted via a small angle MSW mechanism only to the sterile neutrinos. The situation where only a fraction of the missing $\nu_{e} \mathrm{~s}$ convert to $\nu_{s} \mathrm{~s}$ and the rest to active ones has been studied in detail[8] and shown to be quite viable at the moment. The $3+1$ picture on the other hand is severely constrained by the known accelerator data but is also viable for certain values for $\Delta m^{2}$.

If indeed the sterile neutrino turns out to be required, an important theoretical challenge is to seek an understanding of such tiny mass of a particle which is neutral under the standard model forces. This is in clear distinction from the case for the active neutrinos for whom the small mass has a very convincing explanation in terms of the seesaw mechanism 9 that, at the basic level requires only that three singlet right handed neutrinos be introduced with masses close to the GUT scale. The conventional seesaw mechanism makes the three right handed neutrinos superheavy, leaving the three active neutrinos with a very light mass, as seems to be case. In addition to this appealing feature, this minimal seesaw model also restores quark lepton symmetry into the fundamental fermions of nature, making this the standard paradigm of neutrino mass physics. Generic attempts to understand the ultralight sterile neutrino within the seesaw framework involve the introduction of a new singlet fermion into the theory beyond the three right handed neutrinos just discussed and use extra symmetries to protect it from being superheavy. While there exist perfectly acceptable, interesting and technically natural scenarios for the sterile neutrino with extra singlet fermions [10], these extra assumptions have caused skepticism regarding the existence of the sterile neutrino and in turn about the LSND results.

An interesting theoretical challenge is therefore to explore whether within the minimal quark lepton symmetric seesaw framework, one can have an ultralight sterile neutrino without the need for additional singlet neutrinos, outside the quark-lepton symmetric framework and fit all observations including LSND. Such a project is apriori not implausible in view of the fact that the right handed neutrinos already present in the quark-lepton symmetric framework have all the proper- 


\begin{tabular}{|c|c|c|c|c|}
\hline & $S U(2)_{L} \times U(1)_{I_{R}} \times U(1)_{B-L}$ & $S U(2)_{L} \times U_{Y}(1)$ & $L_{e}+L_{\mu}-L_{\tau}$ & $S_{2 R}^{e \mu}$ \\
\hline$\nu_{-R}$ & $(1,1 / 2,-1)$ & $(1,0)$ & 1 & -1 \\
$\nu_{+R}$ & $(1,1 / 2,-1)$ & $(1,0)$ & 1 & 1 \\
$\nu_{\tau R}$ & $(1,1 / 2,-1)$ & $(1,0)$ & -1 & 1 \\
\hline$\Delta$ & $(1,-1,+2)$ & $(1,0)$ & 0 & 1 \\
$\phi$ & $(2,1 / 2,0)$ & $(2,1 / 2)$ & 0 & 1 \\
$\sigma_{2}$ & $(1,0,0)$ & $(1,0)$ & -2 & -1 \\
$\sigma_{0}$ & $(1,0,0)$ & $(1,0)$ & 0 & -1 \\
\hline
\end{tabular}

Table 1: Relevent right handed fermion and scalar fields and their transformation properties. Here we have defined $Y=I_{3 R}+\frac{B-L}{2}$

ties of a sterile neutrino, except the mass. In a recent paper [11] it was shown that if there exist certain leptonic symmetries in the seesaw model, they can make one of the right handed neutrinos massless in the tree approximation of a renormalizable theory making it possible to identify it with the needed ultralight sterile neutrino. Using this idea for the case of an $\left(L_{e}-L_{\mu}-L_{\tau}\right) \times S^{\mu \tau}$ symmetry, (where $S^{\mu \tau}$ is the interchange symmetry between $\nu_{\mu R}$ and $\nu_{\tau R}$ ), it was shown in [11] that the $3+1$ scenario for the LSND observations can be reproduced within the minimal seesaw picture.

In this letter, we show that when the leptonic symmetry is instead chosen to be $\left(L_{e}+L_{\mu}-\right.$ $\left.L_{\tau}\right) \times S_{2}$, the suggestion of ref. 11] can be extended to obtain the $2+2$ explanation of LSND with one of the right handed neutrinos of the seesaw picture playing the role of the sterile neutrino. The mixing of the sterile neutrino to the active neutrinos arises from Planck scale suppressed higher dimensional operators. A consistent picture with $10^{13} \mathrm{GeV}$ for the seesaw scale and $10^{6} \mathrm{GeV}$ for the breaking of the leptonic symmetries seems to emerge and leads to mass matrix ansatz for the $2+2$ case recently proposed in [12]. Experimental tests of this idea are proposed.

\section{Seesaw with a leptonic symmetry and right handed neu- trino as an ultralight sterile neutrino}

In this section, we discuss how imposing $\left(L_{e}+L_{\mu}-L_{\tau}\right) \times S_{e \mu}$ in a seesaw framework leads to a massless right handed neutrino which can play the role of the sterile neutrino. We will work with the gauge group $S U(2)_{L} \times U(1)_{I_{3 R}} \times U(1)_{B-L}$ with particle assignment as in Table I. We call this the minimal seesaw picture since all the fermions in our model are needed in the conventional seesaw explanation of the small masses of the neutrinos. The Higgs field $\Delta^{0}$ transforming as $(1,-1,+2)$ under the gauge group couples to the right handed neutrinos $\left(\nu_{e R}, \nu_{\mu R}, \nu_{\tau R}\right)$ with couplings typically of the form $\nu_{R} \nu_{R} \Delta$. After spontaneous symmetry breaking, the field $\Delta$ acquires a vev i.e. $\left\langle\Delta^{0}\right\rangle=v_{R}$ and breaks the gauge symmetry to the standard model. This gives mass to the right handed neutrinos and is the seesaw scale. The Higgs field $\phi(2,1 / 2,0)$, whose weak scale vev breaks the standard model gauge group, gives Dirac mass to the neutrinos. If we denote the mass matrix for the right handed neutrinos as $M_{R}$, then the complete $6 \times 6$ mass matrix involving 
the Dirac mass and Majorana mass for the neutrinos can be written as

$$
\mathcal{M}_{L R}=\left(\begin{array}{cc}
0 & M_{D} \\
M_{D}^{T} & M_{R}
\end{array}\right) .
$$

When none of the eigenvalues of $M_{R}$ vanishes, one can obtain the mass matrix for the light neutrinos as

$$
\mathcal{M}_{\nu}=-M_{D}^{T} M_{R}^{-1} M_{D}
$$

This is the so-called type I seesaw formula. On the other hand when $M_{R}$ and $M_{D}$ matrices have zero eigenvalues, one must "take them out" of the matrix before using the seesaw formula. As was noted in [11], this turns out to be the case when there are leptonic symmetries. To obtain the $2+2$ scenario, we will consider the leptonic symmetry to be $\left(L_{e}+L_{\mu}-L_{\tau}\right) \times S_{2 R}^{e \mu}$. At the beginning of our analysis we go to a basis where charged lepton mass matrix is diagonal where leptonic mixing matrix is simply neutrino mixing matrix. In this basis we identify the leptonic flavor indices $e, \mu, \tau$. After that the renormalizable Lagrangian invariant under $\left(L_{e}+L_{\mu}-L_{\tau}\right) \times S_{2 R}^{e \mu}$ symmetry has the form

$$
\begin{aligned}
\mathcal{L}_{Y}^{R}= & h_{e} \bar{L}_{e} \phi e_{R}+h_{\mu} \bar{L}_{\mu} \phi \mu_{R}+h_{\tau} \bar{L}_{\tau} \phi \tau_{R} \\
& +h_{1} \bar{L}_{e} \tilde{\phi}\left(\nu_{e R}+\nu_{\mu R}\right)+h_{2} \bar{L}_{\mu} \tilde{\phi}\left(\nu_{e R}+\nu_{\mu R}\right)+h_{3} \bar{L}_{\tau} \tilde{\phi} \nu_{\tau R} \\
& +f\left(\nu_{\mu R}+\nu_{e R}\right) \Delta \nu_{\tau R}
\end{aligned}
$$

Spontaneous symmetry breaking then leads to Dirac and Majorana type mass matrices of the form,

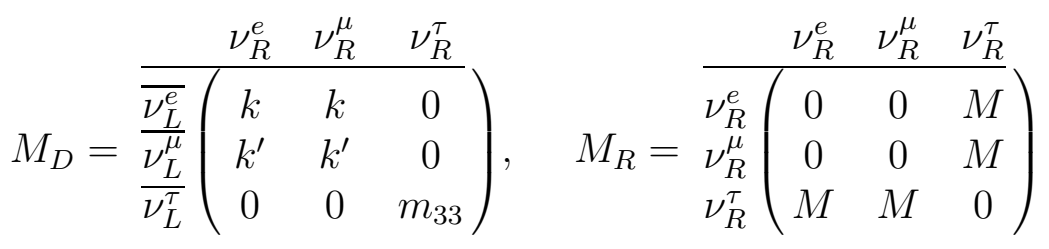

The $6 \times 6$ mass matrix[11] would then take the form

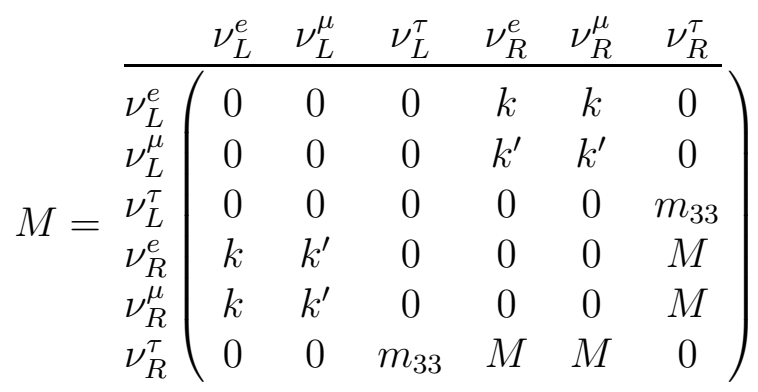


In the rotated basis it is

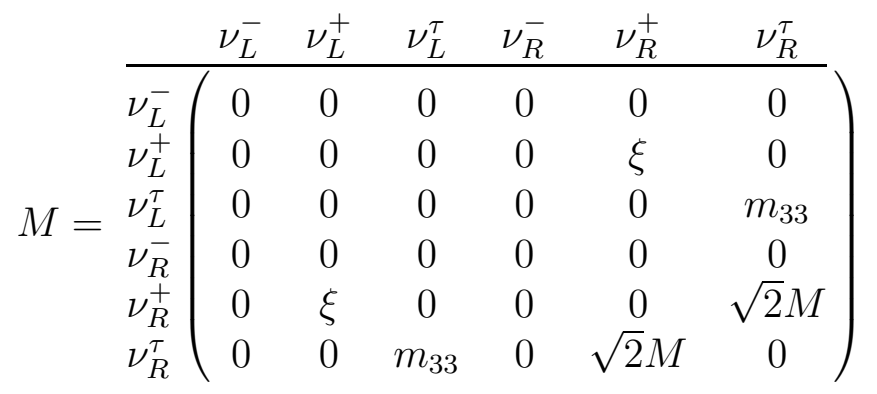

where

$$
\begin{aligned}
\nu_{L}^{-} & =\frac{k^{\prime} \nu_{L}^{e}-k \nu_{L}^{\mu}}{\sqrt{k^{\prime 2}+k^{2}}} \\
\nu_{L}^{+} & =\frac{k \nu_{L}^{e}+k^{\prime} \nu_{L}^{\mu}}{\sqrt{k^{\prime 2}+k^{2}}} \\
\nu_{R}^{ \pm} & =\frac{\nu_{R}^{e} \pm \nu_{R}^{\mu}}{\sqrt{2}}
\end{aligned}
$$

and $\xi=\frac{2 \sqrt{2} k k^{\prime}}{\sqrt{k^{\prime 2}+k^{2}}}$. We next employ the see-saw mechanism to generate the light Majorana neutrino mass matrix,

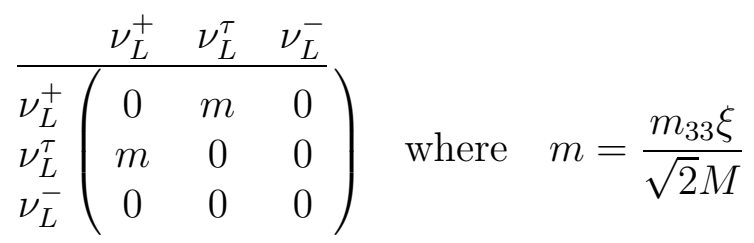

In the limit $k=k^{\prime}$ we have $\xi=2 k$ and $m=\frac{\sqrt{2} k m_{33}}{M}$ [1], however because $S_{2 L}$ symmetry is absent we have the freedom to choose $k \neq k^{\prime}$ which will help to achieve small mixing in the $\nu_{e}-\nu_{\mu}$ sector compatible to LSND.

The challenge after this is to induce large left right mixing. There are higher dimensional operators in this scenario which are sufficient to induce left-right mixing after the see-saw mechanism as will be described below. We can write down following higher dimensional operators

$$
\begin{aligned}
& \frac{f_{1}}{M_{p}} \overline{L_{e}} \nu_{-R}\left\langle\sigma_{0}\right\rangle\langle\tilde{\phi}\rangle \\
& \frac{f_{2}}{M_{p}} \overline{L_{\mu}} \nu_{-R}\left\langle\sigma_{0}\right\rangle\langle\tilde{\phi}\rangle \\
& \frac{f_{3}}{M_{p}} \overline{L_{\tau}} \nu_{-R}\left\langle\sigma_{2}\right\rangle\langle\tilde{\phi}\rangle \\
& \frac{f_{4}}{M_{p}^{2}} \nu_{-R} \nu_{-R}\langle\Delta\rangle\left\langle\sigma_{2}\right\rangle\left\langle\sigma_{0}\right\rangle
\end{aligned}
$$


Now the $4 \times 4$ mass matrix in low energy can be written in terms of rotated low energy fields. We identify $\nu_{L}^{+} \equiv \nu_{e}^{\prime}, \nu_{L}^{-} \equiv \nu_{\mu}^{\prime}, \nu_{-R} \equiv \nu_{s}$

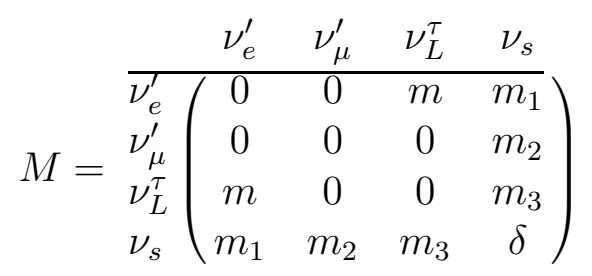

\section{$\underline{\text { CASE } 1}$}

We notice that in the limit $m \rightarrow 0, \delta \rightarrow 0$ the eigenvalues are

$$
0,0, \pm M \quad \text { where } \quad M=\sqrt{m_{1}^{2}+m_{2}^{2}+m_{3}^{2}}
$$

Thus LSND mass splitting squared can be of order $M^{2}$. Introduction of non-zero $m$ and $\delta$ will introduce $\Delta m_{\odot}^{2}$ and $\Delta m_{a t m}^{2}$. To leading order the eigenvectors are,

$$
\begin{aligned}
& \text { Eigenvalue } 0 \quad e_{1}=\left(\begin{array}{c}
-m_{3} / \sqrt{m_{1}^{2}+m_{3}^{2}} \\
0 \\
m_{1} / \sqrt{m_{1}^{2}+m_{3}^{2}} \\
0
\end{array}\right) ; \quad e_{2}=\left(\begin{array}{c}
-m_{1} m_{2} / M \sqrt{m_{1}^{2}+m_{3}^{2}} \\
\sqrt{\left(m_{1}^{2}+m_{3}^{2}\right)} / M \\
-m_{2} m_{3} / M \sqrt{m_{1}^{2}+m_{3}^{2}} \\
0
\end{array}\right) \\
& \text { Eigenvalue } \pm \mathrm{M} \quad e_{3}=\left(\begin{array}{c}
-m_{1} / \sqrt{2} M \\
-m_{2} / \sqrt{2} M \\
-m_{3} / \sqrt{2} M \\
1 / \sqrt{2}
\end{array}\right) ; \quad e_{4}=\left(\begin{array}{c}
m_{1} / \sqrt{2} M \\
m_{2} / \sqrt{2} M \\
m_{3} / \sqrt{2} M \\
1 / \sqrt{2}
\end{array}\right)
\end{aligned}
$$

Depending on the hierarchies between $m_{1}, m_{2}, m_{3}, \delta$ and $m$ various scenarios can emerge from general expressions given in Eqn. (17). Let us consider a simple possibility. Taking $m_{2}>>$ $m_{1}, m_{3}, m, \delta$, and $m_{1} \approx m_{3}$ we have $M=m_{2}$. Eigenvectors reduce to

$$
e_{1}=\left(\begin{array}{c}
-1 / \sqrt{2} \\
0 \\
1 / \sqrt{2} \\
0
\end{array}\right) \quad ; \quad e_{2}=\left(\begin{array}{c}
-1 / \sqrt{2} \\
0 \\
-1 / \sqrt{2} \\
0
\end{array}\right) \quad ; \quad e_{3}=\left(\begin{array}{c}
0 \\
-1 / \sqrt{2} \\
0 \\
1 / \sqrt{2}
\end{array}\right) \quad ; \quad e_{4}=\left(\begin{array}{c}
0 \\
1 / \sqrt{2} \\
0 \\
1 / \sqrt{2}
\end{array}\right)
$$

This means that the massive pair is constituted mainly of $\nu_{\mu}^{\prime}$ and $\nu_{s}$, whereas the light pair is constituted mainly of $\nu_{e}^{\prime}$ and $\nu_{\tau}$ and each pair has large mixing among them. Now take $k>>k^{\prime}$ in Eqn. (7) and Eqn. (8). Then $\nu_{e}^{\prime} \approx \nu_{L}^{e}$ and $\nu_{\mu}^{\prime} \approx \nu_{L}^{\mu}$. We must introduce non-zero $m, \delta m_{1}$ and $m_{2}$ in such a way that the two pairs mix among each other to explain the LSND result.

This can be done numerically for example if we take the parameter set, $m=0.04, m_{1}=$ $0.05, m_{2}=1, m_{3}=0.06, \delta=0.02 \mathrm{eVs}$. We get, $\Delta m_{\odot}^{2}=2 \times 10^{-5}, \Delta m_{\text {atm }}^{2}=4 \times 10^{-2}, \Delta m_{L S N D}^{2}=$ 
$0.98 \mathrm{eV}^{2}$

$$
e_{1}=\left(\begin{array}{c}
0.707 \\
-0.007 \\
-0.706 \\
0
\end{array}\right) e_{2}=\left(\begin{array}{c}
0.705 \\
-0.077 \\
0.704 \\
-0.003
\end{array}\right) e_{3}=\left(\begin{array}{c}
0.033 \\
0.708 \\
0.041 \\
0.703
\end{array}\right) e_{4}=\left(\begin{array}{c}
-0.036 \\
-0.701 \\
-0.044 \\
0.710
\end{array}\right)
$$

We note that now we have introduced small $\nu_{e}-\nu_{\mu}$ mixing even though the primary mixing pattern is governed by Eqn. (18). We can now calculate $\mathrm{P}\left(\overline{\nu_{e}} \rightarrow \overline{\nu_{\mu}}\right)$ for LSND experiment to be

$$
P_{\overline{\nu_{e}} \rightarrow \overline{\nu_{\mu}}}^{L S N D}=4\left|\left(U_{e 3}^{*} U_{\mu 3}+U_{e 4}^{*} U_{\mu 4}\right)\right|^{2} \sin ^{2}\left(\frac{1.27 \Delta m_{L S N D}^{2} L}{E}\right)
$$

On the other hand, we have,

$$
P_{\overline{\nu_{e}} \rightarrow \overline{\nu_{\mu}}}^{L S N}=\sin ^{2} 2 \theta_{L S N D} \sin ^{2}\left(\frac{1.27 \Delta m_{L S N D}^{2} L}{E}\right)
$$

Combining these two equations we have

$$
\sin ^{2} 2 \theta_{L S N D}=0.01
$$

Thus we see that we get $\Delta m^{2}$ as well as $\sin ^{2} 2 \theta$ compatible with LSND experiment 3]. Whereas we have already got large(near-maximal) $\nu_{e} \leftrightarrow \nu_{\tau}$ and large(near-maximal) $\nu_{\mu} \leftrightarrow \nu_{s}$ mixing to account for solar and atmospheric neutrino deficits. However in two-generation analysis of the atmospheric neutrino data the pure $\nu_{\mu} \leftrightarrow \nu_{s}$ oscillation scenario is seen to be highly disfavored, as it fails to reproduce the correct zenith angle distribution of the data, the effect being more for the higher energy neutrinos. The sterile option is also in contradiction with the neutral current sample of the SK atmspheric neutrino data [13]. But from the four-neutrino global analysis of the solar and the atmospheric neutrino data taken together [8] this scenario is not ruled out, although disfavored [8]. The other drawback of this mass texture is that it predicts near-maximal mixing for the solar $\nu_{e} \leftrightarrow \nu_{\tau}$ oscillations. However, after the inclusion of the SNO data, maximal mixing in the LMA region is seen to be disfavored in most analyses of the solar neutrino problem [14].

\section{$\underline{\text { CASE } 2}$}

Let us apply the inverse of the rotation given in Eqn (7) and Eqn (8) on the basis vectors $\left(\nu_{e}^{\prime}, \nu_{\mu}^{\prime}\right)$. Then matrix in Eqn. (15) can be expressed as,

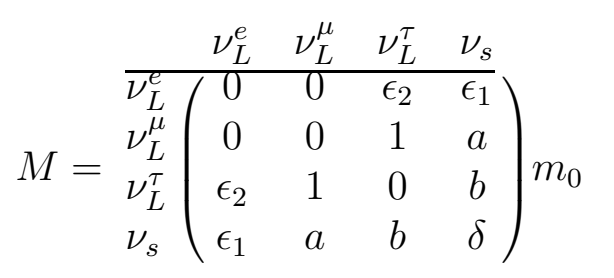

For small b this mass matrix has been studied in Ref. [12 using a different symmetry than the one discussed above. It was shown in detail that it is suitable for the mixed $2+2$ scenario. A similar matrix was also obtained under radiative scheme. However the 44 diagonal element vanish genericaly in simple radiative schemes [15]. 


\section{Predictions of the Model}

With the range of values for the parameter set needed to explain the global solar, atmospheric and the accelerator/reactor data including LSND, our four-generation model makes definite predictions for the effective mass of the neutrinos in beta decay and neutrinoless double beta decay processes. For the neutrinoless double beta decay the "effective Majorana mass parameter" is given by

$$
\begin{aligned}
|<m>| & =\left|\sum_{i=1,4} m_{i} U_{e i}^{2}\right| \\
& =m_{e e}
\end{aligned}
$$

the 11 element of the mass matrix. Since this element is identically zero for the model under consideration, our model predicts no neutrinoless double beta decay. In a recent paper [16] has claimed of a positive evidence for neutrinoless double beta decay, based on the reanalysis of the data from the Heidelberg-Moscow experiment. A best-fit Majorana mass of $0.39 \mathrm{eV}$ for the neutrinos at $95 \%$ confidence level was reported. However there are proposals for new generation neutrinoless double beta decay experiments which are expected to have better sensitivities so they can resolve this issue at higher confidence levels. These include NEMO3 [17, CUORE [18] and GENIUS [19], which hopes to probe neutrino masses down to $\sim 10^{-2} \mathrm{eV}$. This is a good way to falsify the scenario we are presenting here.

The beta decay experiments look for evidence of neutrino mass by observing the end point spectrum of ${ }^{3} H$ beta decay. The best bound available to date is [20, 21]

$$
m_{\nu_{e}}<2.2 \mathrm{eV} \text { at } 95 \% \text { C.L. }
$$

In terms of the mass and mixing angles the quantity probed by the beta decay experiments is

$$
m_{\nu_{e}}^{2}=\sum_{i=1,4} m_{i}^{2}\left|U_{e i}\right|^{2}
$$

For the CASE 2 of our model we have to linear order in $\delta, \epsilon_{1}$ and $\epsilon_{2}$

$$
m_{\nu_{e}}^{2} \approx \frac{m_{0}^{2}}{\left(1+a^{2}\right)}\left[\left(\epsilon_{1}-a \epsilon_{2}\right)^{2}+\left(a \epsilon_{1}+\epsilon_{2}\right)^{2}\right]
$$

With the range of values for the parameters $m_{0}^{2}, a, \epsilon_{1}$ and $\epsilon_{2}$ required to satisfy the global neutrino oscillation data [12] we expect the value of $m_{\nu_{e}} \sim 0.03$ for $\Delta m_{L S N D}^{2} \sim 1 \mathrm{eV}^{2}$. The forthcoming KATRIN experiment is expected to have a sensitivity upto $m_{\nu_{e}} \sim 0.3 \mathrm{eV}$ [22]. Thus we expect no signal for $m_{\nu_{e}}$ in KATRIN from our model. This is another way to test our model.

The other intriguing quantity that needs precise measurements is $\left|U_{e 3}\right|^{2}+\left|U_{e 4}\right|^{2}$ which will give

the fraction of $\nu_{e}$ mixed in the upper doublet. For the CASE 2 upto linear terms in $\delta, \epsilon_{1}$ and $\epsilon_{2}$ we have

$$
\left|U_{e 3}\right|^{2}+\left|U_{e 4}\right|^{2} \approx\left(\frac{a \epsilon_{1}+\epsilon_{2}}{1+a^{2}}\right)^{2}
$$


while

$$
\begin{aligned}
4\left|U_{e 3}^{*} U_{\mu 3}+U_{e 4}^{*} U_{\mu 4}\right|^{2} & \approx 4\left(\frac{a \epsilon_{1}+\epsilon_{2}}{1+a^{2}}\right)^{2} \\
& \approx \sin ^{2} 2 \theta_{L S N D}
\end{aligned}
$$

Thus for the CASE 2 of our model $\left|U_{e 3}\right|^{2}+\left|U_{e 4}\right|^{2} \approx\left(\sin ^{2} 2 \theta_{L S N D}\right) / 4 \approx 0.0007$ much more stringent than the current bound from the short baseline terrestrial experiments (BUGEY 23]) which is

$$
\left|U_{e 3}\right|^{2}+\left|U_{e 4}\right|^{2} \lesssim 0.01
$$

Values of $\left|U_{e 3}\right|^{2}+\left|U_{e 4}\right|^{2}$ as low as 0.0007 would be difficult to detect with usual laboratory beams. However with proposals of high intensity neutrino beams from neutrino factories, it would be interesting to see if one can probe to such accuracy.

\section{Comments and conclusion}

A few comments are now in order: the first theoretical comment is on the choice of the gauge group: while we have illustrated our basic idea in the context of an $S U(2)_{L} \times U(1)_{I_{3 R}} \times U(1)_{B-L}$ gauge group, it could easily be implemented in the context of the standard model gauge group as well. However, in this case the meaning of the seesaw scale will remain a mystery. One could also use the left-right symmetric gauge group $S U(2)_{L} \times S U(2)_{R} \times U(1)_{B-L} 24$. In this case since the right handed neutrino and the right handed charged leptons are in the same multiplet, $S_{e \mu}$ symmetry leads to electron being mass less prior to symmetry breaking. One can however extend the model to make the electron massive. Quark lepton unification of these models is nontrivial due to absence of any analog of $L_{e}+L_{\mu}-L_{\tau}$ symmetry in the quark sector.

The breaking of the leptonic symmetries can be made soft by adding to the Lagrangian terms such as $\left(\sigma_{2}^{-}\right)^{2}$ so that there are no massless scalar bosons.

In conclusion, we have presented a minimal seesaw model using three active and three right handed neutrinos (as would be suggested in a quark-lepton symmetric theory), which can lead to an ultralight sterile neutrino. The sterile neutrino in this case is none other than the right handed neutrino. Spontaneous breaking of the leptonic symmetries lead to mixing of the sterile neutrino with the the active neutrinos resulting in a $2+2$ hybrid scenario for LSND, which provides a viable description of neutrino oscillation data. The model predicts an effective $U_{e 3} \simeq 0.025$ and an effective end point mass in tritium beta decay $\simeq 0.03$.

The work of RNM is supported by the National Science Foundation Grant No. PHY-0099544. B.B and S.C would like to thank the organizers of WHEPP-7 where a part of this work was done. B.B would also like to thank Amitava Raychaudhuri and Probir Roy for discussions on Ref. [11].

\section{References}

[1] Y. Suzuki et al., Super-Kamiokande collaboration, Nucl. Phys. Proc. Suppl. 77, 35 (1999); B. Cleveland et al., Ap. J. 496, 505 (1998);J. N. Abduratshitov et al., SAGE collaboration, 
Phys. Rev. C 60, 055801 (1999); W. Hampel et al., GALLEX collaboration, Phys. Lett. B447, 127 (1999); M. Altman et al., GNO collaboration, Phys. Lett. B490, 16 (2000); Q. Ahmed et al, SNO collaboration, nucl-ex/0106015.

[2] Y. Fukuda et al., Phys. Rev. Lett. 81, 1562 (1998); R. Becker-Szendy et al., Phys. Rev. D 46, 3720 (1992); S. H. Hirata et al., Phys. Lett. B 280, 146 (1992); W. W. M. Allison et al., Phys. Lett. B 449, 137 (1999); M. Ambrosio et al., Phys. Lett. B 517, 59 (2001).

[3] C. Athanassopoulos et al., Phys. Rev. C54 (1996) 2685; C. Athanassopoulos et al.,, Phys. Rev. C58, 2489 (1998); A. Aguilar et al., hep-ex/0104049.

[4] K. Eitel et al., KARMEN collaboration, hep-ex/0008002.

[5] See talk by J. Conrad, WIN2002 workshop in Christ Church, Newzealand (January, 2002).

[6] D. O. Caldwell and R. N. Mohapatra, Phys. Rev. D 48, 3259 (1993); J. Peltoniemi and J. W. F. Valle, Nucl. Phys. B406, 409 (1993); J. Peltoniemi, D. Tomassini and J. W. F. Valle, Phys. Lett. B 298, 383 (1993); for detailed analyses and other references, see S. M. Bilenky, C. Giunti and W. Grimus, Prog. Part. Nucl. Phys. 43, 1 (1999); N. Okada and O. Yasuda, Int. J. Mod. Phys. A 12, 3669 (1997).

[7] S. M. Bilenky, C. Giunti, W. Grimus and T. Schwetz, Phys. Rev. D60, 073007 (1999); B. Balatenkin, G. Fuller, J. Fetter and G. McGlaughlin, Phys. Rev. C 59, 2873 (1999); V. Barger, B. Kayser, J. Learned, T. Weiler, K. Whisnant, Phys. Lett. B489, 345 (2000); O. Peres and A. Y. Smirnov, hep-ph/0011054; K. Balaji, A. Perez-Lorenzana and A. Smirnov, hep-ph/0101005; R. N. Mohapatra, hep-ph/0107274; Phys. Rev. D 64, 091301; K. S. Babu and R. N. Mohapatra, hep-ph/0201776.

[8] M. C. Gonzales-Garcia, M. Maltoni and C. Pena-Garay, hep-ph/0108073; M. Maltoni, T. Schwetz, J.W.F. Valle, e-Print Archive: hep-ph/0112103.

[9] M. Gell-Mann, P. Ramond and R. Slansky, in Supergravity, eds. P. van Niewenhuizen and D.Z. Freedman (North Holland 1979); T. Yanagida, in Proceedings of Workshop on Unified Theory and Baryon number in the Universe, eds. O. Sawada and A. Sugamoto (KEK 1979); R.N. Mohapatra and G. Senjanović, Phys. Rev. Lett. 44, 912 (1980).

[10] R. Foot and R. Volkas, Phys. Rev. D 52, 6595 (1995); Z. Berezhiani and R. N. Mohapatra, Phys. Rev. D 52, 6607 (1995); E. Ma and P. Roy, Phys. Rev. D 52, 4342 (1995); K. Benakli and A. Smirnov, Phys. Rev. Lett. 79, 4314 (1997); P. Langacker, hep-ph/9805281; D. Suematsu, Phys. Lett. B392, 413 (1997); E. J. Chun, A. Joshipura and A. Smirnov, Phys. Rev. D 54, 4654 (1996); E. Ma, Phys. Lett. B 30, 286 (1996); Z. Chacko and R. N. Mohapatra, hep-ph/9905388.

[11] R.N. Mohapatra, Phys. Rev. D64 091301 (2001)

[12] K.S. Babu, R.N. Mohapatra, Phys. Lett. B522 287 (2001) 
[13] The Super-Kamiokande Collaboration, S. Fukuda et al., Phys. Rev. Lett. 85, 3999 (2000).

[14] A. Bandyopadhyay, S. Choubey, S. Goswami , K. Kar, Phys. Lett. B519, 83 (2001); G.L. Fogli, E. Lisi, D. Montanino, A. Palazzo, Phys. Rev. D64, 093007 (2001); J.N. Bahcall, M.C. Gonzalez-Garcia, C. Pana-Garay, JHEP 0108, 014 (2001); P.I. Krastev and A.Yu. Smirnov, e-Print Archive: hep-ph/0108177; M.V. Garzelli and C. Giunti, JHEP 0112, 017 (2001).

[15] S. Goswami and A. Joshipura, e-Print Archive: hep-ph/011027; P. Roy, S. K. Vempati, Phys. Rev. D65, 073011 (2002).

[16] H.V. Klapdor-Kleingrothaus et al., Mod. Phys. Lett. A37, 2409 (2001).

[17] C. Marquet et al., Nucl. Phys. Proc. Suppl. 87, 298 (2000).

[18] E. Fiorini, Phys. Rep. 307, 309 (1998).

[19] H.V. Klapdor-Kleingrothaus, J. Hellmig and M. Hirsch, J. Phys. G24, 483 (1998); L. Baudis et al., Phys. Rep. bf 307, 301 (1998).

[20] The MAINZ Collaboration, J. Bonn et al., Nucl. Phys. Proc. Suppl. 91, 273 (2001).

[21] The TROITSK Collaboration, V.M. Lobashev et al., Nucl. Phys. Proc. Suppl. 91, 280 (2001).

[22] The KATRIN Collaboration, A. Osipowicz et al., hep-ex/0109033.

[23] B. Achkar et al., Nucl. Phys. B424, 503 (1995).

[24] J. C. Pati and A. Salam, Phys. Rev. D10, 275 (1974); R. N. Mohapatra and J. C. Pati, Phys. Rev. D 11, 566 (1975); Phys. Rev. D 11, 2558 (1975); G. Senjanović and R. N. Mohapatra, Phys. Rev. D 12, 1502 (1975). 